International Journal of Pure and Applied Mathematics

Volume 102 No. 4 2015, 613-630

ISSN: 1311-8080 (printed version); ISSN: 1314-3395 (on-line version)

url: http://www.ijpam.eu

doi: http://dx.doi.org/10.12732/ijpam.v102i4.3

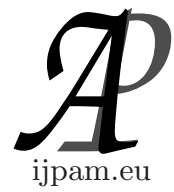

\title{
SOME PROPERTIES OF $\alpha$-OPEN SETS WITH RESPECT TO AN IDEAL
}

\author{
A.A. $\operatorname{Nasef}^{1}$, A.E. Radwan ${ }^{2}$, R.B. Esmaeel ${ }^{3} \S$ \\ ${ }^{1}$ Department of Physics and Engineering Mathematics \\ Faculty of Engineering \\ Kafr El-Sheikh University \\ Kafr El-Sheikh, EGYPT \\ ${ }^{2}$ Department of Mathematics \\ Faculty of Science \\ Ain Shams University \\ EGYPT \\ ${ }^{3}$ Department of Mathematics \\ Ibn Al-Haitham College of Education \\ Baghdad University \\ IRAQ
}

\begin{abstract}
The aim of this paper is to introduce some types of compactness and connectedness in ideal topological spaces. We further study some properties of these types. Finally, the relationship between various types of compactness and connectedness was summarized.
\end{abstract}

AMS Subject Classification: 54A05, 54C10, 54 D05, 54D30

Key Words: $\quad \alpha_{I}$-open sets, $w \cdot \alpha I$-open sets, $w \cdot I \alpha$-open sets, $c \alpha_{I}$-compact, $c w \cdot \alpha I$-compact, $c w \cdot I \alpha$-compact, $\alpha_{I}$-separated connected, $w \cdot \alpha I$-separated connected, $w \cdot I \alpha$-separated connected, ideal

\section{Introduction and Preliminaries}

Throughout the present paper, $(X, \tau),(Y, \sigma)$ and $(Z, \mu)$ will denote topological spaces with no separation properties assumed. For a subset $A$ of a topological

Received: November 16, 2014

(c) 2015 Academic Publications, Ltd.

${ }^{\S}$ Correspondence author url: www.acadpubl.eu 
space $(X, \tau), \operatorname{cl}(A)$ and $\operatorname{int}(A)$ will denote the closure and interior of $A$ in $(X, \tau)$, respectively.

The subject of ideals in topological spaces has been studied by Kuratowski [8] and Vaidyanathaswamy [14]. An ideal $I$ on a set $X$ is a nonempty collection of subsets of $X$ which satisfies the conditions:

(1) $A \in I$ and $B \subseteq A$ implies $B \in I$,

(2) $A \in I$ and $B \in I$ implies $A \cup B \in I$.

Given a topological space $(X, \tau)$ with an ideal $I$ on $X$ and $P(X)$ is the set of all subsets of $X$. A set operator ()$^{*}: P(X) \rightarrow P(X)$ is called a local function [14] of $A$ with respect to $\tau$ and $I$ iff for $A \subseteq X, A^{*}(I, \tau)=\{x \in X: A \cap U \notin$ $I$ for every $U \in \tau(x)\}$ where $\tau(x)=\{U \in \tau: x \in U\}$. A Kuratowski closure operator $c l^{*}(\quad)$ [14] for a topology $\tau^{*}(I, \tau)$, called the $*$-topology, finer than $\tau$ is defined by $c l^{*}(A)=A \cup A^{*}(I, \tau)$. When there is no chance for confusion, we will simply write $A^{*}$ for $A^{*}(I, \tau)$ and $\tau^{*}$ for $\tau^{*}(I, \tau)$. If $I$ is an ideal on $X$, then $(X, \tau, I)$ is called an ideal topological space.

In an ideal topological space $(X, \tau, I)$, if $A \subseteq X$, int* $(A)$ will denote the interior of $A$ in $\left(X, \tau^{*}\right)$. Closed subsets in $\left(X, \tau^{*}\right)$ are called $\tau^{*}$-closed sets. A subset $A$ of an ideal topological space $(X, \tau, I)$ is $\tau^{*}$-closed [14] if and only if $A^{*} \subseteq A$.

For any ideal topological space $(X, \tau, I)$, the collection $\{V-J: V \in$ $\tau$ and $J \in I\}$ is a basis for $\tau^{*}$. The elements of $\tau^{*}$ are called $\tau^{*}$-open sets. A subset $A$ of an ideal topological space $(X, \tau, I)$ is said to be $\tau^{*}$-dense if $c l^{*}(A)=X$. It is clear that, in a space $(X, \tau, I)$ if $I=\{\phi\}$, then $\tau=\tau^{*}[4],[7]$.

Recall that if $(X, \tau, I)$ is an ideal topological space and $A$ is a subset of $X$, then $\left(A, \tau_{A}, I_{A}\right)$ is an ideal topological space where $\tau_{A}$ is the relative topology on $A$ and $I_{A}=\{A \cap J: J \in I\}$.

It is interesting to note that if $I$ is a proper ideal (i.e. $X \notin I$ ), then the collection of the complements of the members of $I$ form a filter on $X$. This is why sometimes ideals are called dual filters.

The following collections form important ideals [7] on a topological space $(X, \tau)$ :

(i) $\{\phi\}$ or $I_{\{\phi\}}$ : the trivial ideal.

(ii) $P(X)$ : the improper ideal.

(iii) $F:\left(I_{f}\right)$ the ideal of all finite sets.

(iv) $C:\left(I_{c}\right)$ the ideal of all countable subsets of $X$. 
A subset $A$ of a topological space $(X, \tau)$ is said to be $\alpha$-open [11] if $A \subseteq$ $\operatorname{int}(\operatorname{cl}(\operatorname{int}(A)))$. The complement of a $\alpha$-open set in a space $(X, \tau)$ is said to be $\alpha$-closed [11]. The family of all $\alpha$-open sets in a topological space $(X, \tau)$ is a topology on $X$ finer than $\tau$ denoted by $\tau_{\alpha}$. A topological space $(X, \tau)$ is said to be $\alpha$-compact [9] if every cover of $X$ by $\alpha$-open sets has a finite subcover.

A subset $A$ of an ideal topological space $(X, \tau, I)$ is said to be $I$-compact [13] if for every cover $\left\{U_{\lambda}: \lambda \in \Lambda\right\}$ of $A$ by open sets of $X$, there exists a finite subset $\Lambda_{0}$ of $\Lambda$ such that $A-\cup\left\{U_{\lambda}: \lambda \in \Lambda_{0}\right\} \in I$. An ideal topological space $(X, \tau, I)$ is said to be $I$-compact if $X$ is $I$-compact as a subset.

The concept of a set operator ()$^{\alpha^{*}}: P(X) \rightarrow P(X)$ introduced by A. A. Nasef in 1992 [10] and called an $\alpha$-local function of $I$ with respect to $\tau$ and defined as follows: for $A \subseteq X, A^{\alpha^{*}}(I, \tau)=\{x \in X: A \cap U \notin I$ for every $U \in$ $\left.\tau_{\alpha}(x)\right\}$ where $\tau_{\alpha}(x)=\left\{U \in \tau_{\alpha}: x \in U\right\}$. When there is no ambiguity, we will simply write $A^{\alpha^{*}}$ for $A^{\alpha^{*}}(I, \tau)$. A $\tau^{\alpha^{*}}$-closure operator, denoted by $\mathrm{cl}^{\alpha^{*}}(\quad)$. for a topology $\tau^{\alpha^{*}}(I)$ which is called $\tau^{\alpha^{*}}$-topology on $X$ finer than $\tau$ is defined as follows: $c l^{\alpha^{*}}(A)(I, \tau)=A \cup A^{\alpha^{*}}(I, \tau)$ for every $A \subseteq X$. When there is no ambiguity, we will simply write $c l^{\alpha^{*}}(A)$ for $c l^{\alpha^{*}}(A)(I, \tau)$. A basis $\mathcal{B}(I, \tau)$ for $\tau^{\alpha^{*}}$ was described as follows: $\mathcal{B}(I, \tau)=\left\{V-J: V \in \tau_{\alpha}\right.$ and $\left.J \in I\right\}$. We will denote by $i n t^{\alpha^{*}}(A)$ and $c l^{\alpha^{*}}(A)$ the interior and closure of $A \subseteq X$, with respect to $\tau^{\alpha^{*}}$. The elements of $\tau^{\alpha^{*}}$ are called $\tau^{\alpha^{*}}$-open sets. Closed subsets in $\left(X, \tau^{\alpha^{*}}\right)$ are called $\tau^{\alpha^{*}}$-closed set. A subset $A$ of an ideal topological space $(X, \tau, I)$ is $\tau^{\alpha^{*}}$-closed (resp., $\tau^{\alpha^{*}}$-dense) if and only if $A^{\alpha^{*}} \subseteq A$ (resp., $\left.c l^{\alpha^{*}}(A)=X\right)$.

A subset $A$ of an ideal topological space $(X, \tau, I)$ is said to be $\alpha I$-open [6] (resp., $I \alpha$-open [3]) if $A \subseteq \operatorname{int}\left(c l^{*}(\operatorname{int}(A))\right)$ (resp., $\left.A \subseteq \operatorname{int}\left(c l^{\alpha^{*}}(\operatorname{int}(A))\right)\right)$. The complement of an $\alpha I$-open (resp. $I \alpha$-open) set in a space $(X, \tau)$ is said to be $\alpha I$-closed (resp. $I \alpha$-closed) . We will denote the family of all $\alpha I$-open (resp., $I \alpha$-open) sets by $\alpha I O(X)$ (resp., $I \alpha O(X))$.

\section{2. $\alpha$-Compactness with Respect to an Ideal}

In this section, we will introduce new types of compactness in an ideal topological space by using the concepts of $\alpha_{I}$ open, $w \cdot \alpha I$-open and $w \cdot I \alpha$ - open sets. Some properties of these types are studied.

\section{Definition 1. [1]}

A subset $A$ of an ideal topological space $(X, \tau, I)$ is said to be

(i) $\alpha$-open set with respect to $I$ (simply, $\alpha_{I}$-open) iff there exists an open set $U$ such that $U-A \in I$ and $A-\operatorname{int}(\operatorname{cl}(U)) \in I$. 
(ii) weakly $\alpha I$-open set (simply, w. $\alpha I$-open) iff there exists an open set $U$ such that $U-A \in I$ and $A-\operatorname{int}\left(c l^{*}(U)\right) \in I$.

(iii) weakly $I \alpha$-open set (simply, w.I $\alpha$-open) iff there exists an open set $U$ such that $U-A \in I$ and $A-\operatorname{int}\left(c l^{\alpha^{*}}(U)\right) \in I$.

The family of all $\alpha_{I}$-open (resp., $w . \alpha I$-open and $w . I \alpha$-open) sets is denoted by $\alpha_{I} O(X)$ (resp., w. $\alpha I O(X)$ and $\left.w . I \alpha O(X)\right)$. The complement of an $\alpha_{I}$-open (resp., $w . \alpha I$-open and $w . I \alpha$-open) set is said to be $\alpha_{I}$-closed (resp., $w . \alpha I$-closed and $w . I \alpha$-closed) set. The family of all $\alpha_{I}$-closed (resp., $w . \alpha I$-closed and $w . I \alpha$ closed) sets is denoted by $\alpha_{I} C(X)$ (resp., w. $\alpha I C(X)$ and $\left.w \cdot I \alpha C(X)\right)$.

It is clear that every $\alpha$-open (resp. $\alpha I$-open and $I \alpha$-open) set is $\alpha_{I}$-open (resp. $w . \alpha I$-open and $w . I \alpha$-open). If $I=\{\phi\}$, then the two concepts $\alpha$ openness (resp. $\alpha I$-openness and $I \alpha$-openness) and $\alpha_{I}$-openness (resp. $w . \alpha I$ openness and $w . I \alpha$-openness) are equivalent. So, if $A \in I$, then both $A$ and $X-A$ are $\alpha_{I}$-open (resp. $w . \alpha I$-open and $w . I \alpha$-open) sets. In a special case, if $\tau$ is the discrete (resp. indiscrete) topology on a set $X$ and $I=\{\phi\}$ then the concepts of openness, $\alpha$-openness, $\alpha_{I}$-openness, $\alpha I$-openness, $w . \alpha I$-openness, $I \alpha$-openness and $w . I \alpha$-openness are equivalent [1].

The following diagram shows the relations between the different types of weakly open sets that studied in this article.

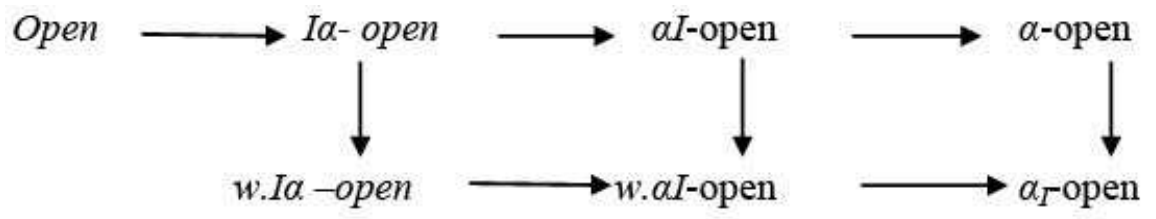

The opposite direction of the implication in the above diagram may be false [1].

Definition 2. A subset $A$ of an ideal topological space $(X, \tau, I)$ is said to be $\alpha_{I^{-}}$compact (resp. $\alpha I$ - compact, $I \alpha$ - compact, $w \cdot \alpha I$ - compact and $w . I \alpha$ compact) if every cover of $A$ by $\alpha_{I^{-}}$open (resp. $\alpha I$-open, $I \alpha$-open, $w . \alpha I$ - open and $w . I \alpha$-open) sets in $X$ has a finite subcover. A space $X$ is said to be $\alpha_{I^{-}}$ compact (resp. $\alpha I$ - compact, $I \alpha$-compact, $w \cdot \alpha I$ - compact and $w . I \alpha$ - compact) space if it is an $\alpha_{I}$ - compact (resp. $\alpha I$-compact, I $\alpha$ - compact, $w . \alpha I$ - compact and $w . I \alpha$ - compact) as a subset.

The following diagram shows the relations between the different types of compactness which introduced in Definition 2; 

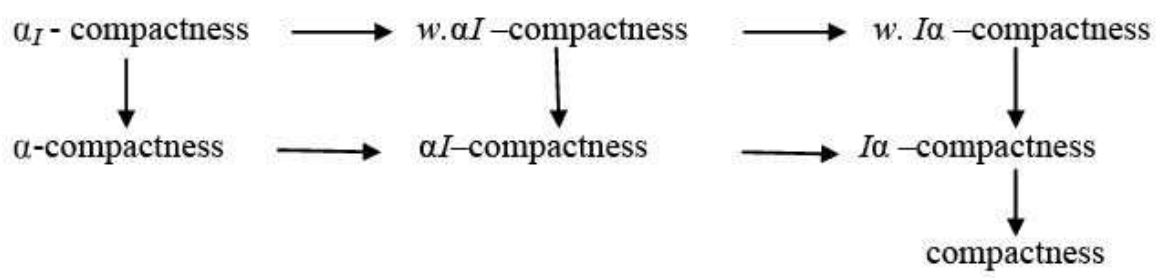

The opposite direction of the implications in the above diagram may be false. For examples:

Example 3. Consider the ideal topological space $(\mathbb{N}, \tau, I)$, where $\mathbb{N}$ is the set of all natural numbers, $\tau=\{\mathbb{N}, \phi,\{1\}\}$ and $I=\{\phi\}$. It is clear that $(\mathbb{N}, \tau)$ is a compact space but $(\mathbb{N}, \tau, I)$ is not $I \alpha$-compact. Since, $\{\{1, n\}: n \in \mathbb{N}\}$ is an $I \alpha$-open cover for $\mathbb{N}$ which has no finite subcover.

Example 4. Consider the ideal topological space $(\mathbb{N}, \tau, I)$, where $\mathbb{N}$ is the set of all natural numbers, $\tau=\{\mathbb{N}, \phi,\{I\}\}$ and $I=\{\phi,\{1\}\}$. It is clear that $(\mathbb{N}, \tau, I)$ is an $\alpha I$-compact space but $(\mathbb{N}, \tau)$ is not $\alpha$-compact. Since, $\{\{1, n\}$ : $n \in \mathbb{N}\}$ is an $\alpha$-open cover for $\mathbb{N}$ which has no finite subcover.

Similarly, $(\mathbb{N}, \tau, I)$ is $w . \alpha I$-compact space which is not $\alpha_{I}$-compact.

Example 5. Consider the ideal topological space $(\mathbb{N}, \tau, I)$, where $\mathbb{N}$ is the set of all natural numbers, $\tau=\{\mathbb{N}, \phi\}$ and $I=P(\mathbb{N})$. It is clear that $(\mathbb{N}, \tau)$ is an $\alpha$-compact space but $(\mathbb{N}, \tau, I)$ is not $\alpha_{I}$-compact. Since, $\{\{n\}: n \in \mathbb{N}\}$ is an $\alpha_{I}$-open cover for $\mathbb{N}$ which has no finite subcover.

Similarly, ( $\mathbb{N}, \tau, I)$ is an $\alpha I$-compact (resp. I $\alpha$-compact) space which is not $w . \alpha I$-compact (resp. w.I $\alpha$-compact) .

Example 6. Consider the ideal topological space $(\mathbb{N}, \tau, I)$, where $\mathbb{N}$ is the set of all natural numbers, $\tau=\{\mathbb{N}, \phi,\{1\},\{1,2\}\}$ and $I=\{\phi,\{1\}\}$. It is clear that $(\mathbb{N}, \tau, I)$ is an $I \alpha$-compact (resp. w.I $\alpha$-compact) space which is not $\alpha I$ compact (resp. not w. $\alpha I$-compact). Since, $\{\{1,2, n\}: n \in \mathbb{N}\}$ is an $I \alpha$-open (resp. w.I $\alpha$-open) cover for $\mathbb{N}$ which has no finite subcover.

Definition 7. A subset $A$ of an ideal topological space $(X, \tau, I)$ is said to be compatible $\alpha$-compact (resp. compatible $\alpha_{I}$-compact, compatible $\alpha I$ compact, compatible $I \alpha$-compact, compatible $w \cdot \alpha I$-compact and compatible $w . I \alpha$-compact) with respect to $I$, simply $c \alpha$-compact (resp. $c \alpha_{I}$-compact, $c \alpha I$ compact, $c I \alpha$-compact, $c w \cdot \alpha I$-compact and $c w \cdot I \alpha$-compact) if for every cover $\left\{U_{\lambda}: \lambda \in \Lambda\right\}$ of $A$ by $\alpha$-open (resp. $\alpha_{I}$-open, $\alpha I$-open, $I \alpha$-open, $w \cdot \alpha I$-open and $w . I \alpha$-open) sets in $X$, there exists a finite subset $\Lambda_{0}$ of $\Lambda$ such that $A-\cup\left\{U_{\lambda}\right.$ : 
$\left.\lambda \in \Lambda_{0}\right\} \in I$. An ideal topological space $(X, \tau, I)$ is said to be $c \alpha$-compact (resp. c $\alpha_{I}$-compact, $c \alpha I$-compact, $c I \alpha$-compact, $c w \cdot \alpha I$-compact and $c w . I \alpha-$ compact) if $X$ is $c \alpha$-compact (resp. $c \alpha_{I}$-compact, $c \alpha I$-compact, $c I \alpha$-compact, $c w . \alpha I$-compact and $c w . I \alpha$-compact) as a subset.

The following diagram shows the relations between the different types of compactness which introduced in Definition 7;

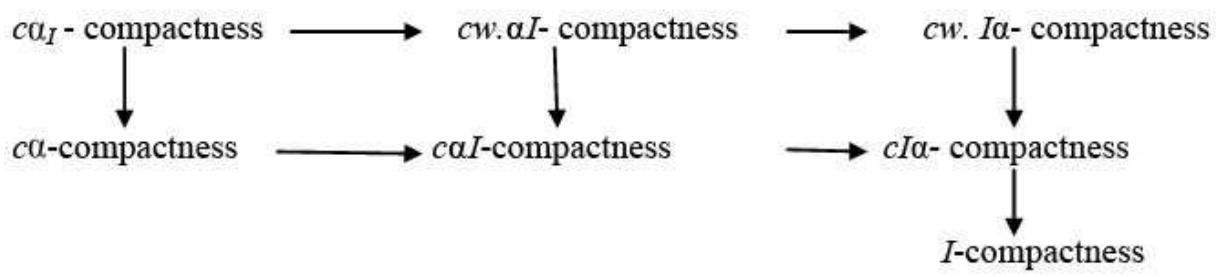

The opposite direction of the implications in the above diagram may be false. For examples:

Example 8. Consider the ideal topological space $(\mathbb{N}, \tau, I)$, where $\mathbb{N}$ is the set of all natural numbers, $\tau=\{\mathbb{N}, \phi\}$ and $I=I_{f}=\{U \subseteq \mathbb{N}: U$ is a finite set $\}$. It is clear that $(\mathbb{N}, \tau, I)$ is $c \alpha$-compact (resp. c $\alpha I$-compact and $c I \alpha$-compact) space which is not $c \alpha_{I}$-compact (resp. not $c w . \alpha I$-compact and not $c w . I \alpha-$ compact). Since, $\{\{n\}: n \in \mathbb{N}\}$ is an $\alpha_{I}$-open (resp. w. $\alpha I$-open and w.I $\alpha$ open) cover for $\mathbb{N}$ which has no finite subfamily $\{\{n\}: n=1,2, \cdots, r\}$ such that $\mathbb{N}-\cup\{\{n\}: n=1,2, \cdots, r\} \in I$.

Now, we can see that the ideal topological space $(\mathbb{N}, \tau, I)$ in Example 3 is an $I$-compact space which is not $c I \alpha$-compact. So, the ideal topological space $(\mathbb{N}, \tau, I)$ in Example 4 is $c \alpha I$-compact (resp. cw. $\alpha I$-compact) which is not $c \alpha$ compact (resp. not $c \alpha_{I}$-compact) . Also, the ideal topological space $(\mathbb{N}, \tau, I)$ in Example 6 is $c I \alpha$-compact (resp. cw.I $\alpha$-compact) which is not $c \alpha I$-compact (resp. not $c w . \alpha I$-compact) .

Remark 9. Every $\alpha_{I}$-compact (resp. $\alpha I$-compact, $I \alpha$-compact, $w . \alpha I$ compact, $w . I \alpha$-compact and $\alpha$-compact) space is $c \alpha_{I}$-compact (resp. $c \alpha I$ compact, $c I \alpha$-compact, $c w \cdot \alpha I$-compact, $c w . I \alpha$-compact and $c \alpha$-compact) . Not conversely, for example;

Example 10. Consider the ideal topological space $(\mathbb{N}, \tau, I)$, where $\mathbb{N}$ is the set of all natural numbers, $\tau=I=P(\mathbb{N})$. It is clear that $(\mathbb{N}, \tau, I)$ is $c \alpha_{I^{-}}$ compact (resp. c $\alpha I$-compact, $c I \alpha$-compact, cw. $\alpha I$-compact, $c w . I \alpha$-compact 


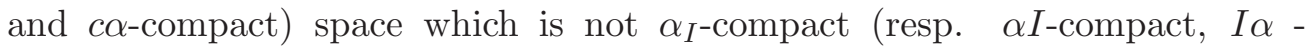
compact, $w \cdot \alpha I$-compact, $w \cdot I \alpha$-compact and $\alpha$-compact) .

Proposition 11. For an ideal topological space $(X, \tau, I)$, the following statements are equivalent;

(i) $(X, \tau)$ is $\alpha$ - compact.

(ii) $(X, \tau,\{\phi\})$ is $\alpha_{I^{-}}$compact.

(iii) $(X, \tau,\{\phi\})$ is $\alpha I$ - compact.

(iv) $(X, \tau,\{\phi\})$ is $w . \alpha I$ - compact.

(v) $(X, \tau)$ is c $\alpha$ - compact.

(vi) $(X, \tau,\{\phi\})$ is $c \alpha_{I^{-}}$compact.

(vii) $(X, \tau,\{\phi\})$ is coI- compact.

(viii) $(X, \tau,\{\phi\})$ is cw. $\alpha I$ - compact.

Proposition 12. For an ideal topological space $(X, \tau, I)$, the following statements are equivalent;

(i) $(X, \tau,\{\phi\})$ is I $\alpha$ - compact.

(ii) $(X, \tau,\{\phi\})$ is $w \cdot I \alpha$ - compact.

(iii) $(X, \tau,\{\phi\})$ is $c I \alpha$ - compact.

(iv) $(X, \tau,\{\phi\})$ is cw.I $\alpha$ - compact.

In the following proposition, we will give a characterization for $\alpha_{I^{-}}$compactness.

Proposition 13. In an ideal topological space $(X, \tau, I)$, the following statements are equivalent;

(i) $(X, \tau, I)$ is $\alpha_{I^{-}}$compact.

(ii) For any family $\left\{F_{\lambda}: \lambda \in \Lambda\right\}$ of $\alpha_{I^{-}}$closed sets of $X$ such that $\cap\left\{F_{\lambda}: \lambda \in\right.$ $\Lambda\}=\phi$, there exists a finite subset $\Lambda_{0}$ of $\Lambda$ such that $\cap\left\{F_{\lambda}: \lambda \in \Lambda_{0}\right\}=\phi$.

In similar way, we can give a characterization for $\alpha_{I}$-compactness (resp. $I \alpha$-compactness, $w . \alpha I$ compactness and $w . I \alpha$-compactness) .

In the following proposition, we will give a characterization for $c \alpha_{I^{-}}$compactness. 
Proposition 14. In an ideal topological space $(X, \tau, I)$, the following statements are equivalent;

(i) $(X, \tau, I)$ is $c \alpha_{I^{-}}$compact.

(ii) For any family $\left\{F_{\lambda}: \lambda \in \Lambda\right\}$ of $\alpha_{I^{-}}$closed sets of $X$ such that $\cap\left\{F_{\lambda}: \lambda \in\right.$ $\Lambda\}=\phi$, there exists a finite subset $\Lambda_{0}$ of $\Lambda$ such that $\cap\left\{F_{\lambda}: \lambda \in \Lambda_{0}\right\} \in I$.

In similar way, we can give a characterization for $c \alpha I$-compactness (resp. $c I \alpha$-compactness, $c w . \alpha I$-compactness, $c w . I \alpha$-compactness and $c \alpha$-compactness)

Proposition 15. In an ideal topological space $(X, \tau, I)$, if $I=\{\phi\}$ and every nowhere dense set is closed, then all concepts of compactness which are studied in this article are equivalent.

Remark 16. If $I$ and $J$ are two ideals on a topological space $(X, \tau)$, with $I \subseteq J$, then:

(i) The condition $(X, \tau, I)$ is $c \alpha_{I}$-compact (resp. cw. $\alpha I$-compact and $c w \cdot I \alpha$ -compact) not sufficient to make $(X, \tau, J)$ is $c \alpha_{J}$-compact (resp. cw. $\alpha J$ compact and cw.J $\alpha$-compact) . For example; Consider the topological space $(\mathbb{N}, \tau)$ where $\tau$ is the indiscrete topology on $\mathbb{N}$, with two ideals $I=\{\phi\}$ and $J=J_{f}$ is the ideal of all finite subsets of $\mathbb{N}$.

It is clear that $(\mathbb{N}, \tau, I)$ is $c \alpha_{I}$-compact (resp. $c w . \alpha I$-compact and $c w . I \alpha$ compact) but $(\mathbb{N}, \tau, J)$ is not $c \alpha_{J}$-compact (resp. not cw. $\alpha J$-compact and not $c w . J \alpha$-compact), since $\{\{n\}: n \in \mathbb{N}\}$ is $\alpha_{J}$-open (resp. w. $\alpha J$-open and $w . J \alpha$-open) cover for $\mathbb{N}$ which has no finite subfamily $\{\{n\}: n=$ $1,2, \cdots, r\}$ such that $\mathbb{N}-\cup\{\{n\}: n=1,2, \cdots, r\} \in J$.

(ii) The condition $(X, \tau, J)$ is $c \alpha_{J}$-compact (resp. cw. $\alpha J$-compact and $c w . J \alpha$ -compact) is not sufficient to make $(X, \tau, I)$ is $c \alpha_{I}$-compact (resp. $c w . \alpha I$ compact and $c w . I \alpha$-compact). For example; Consider the topological space $(\mathbb{N}, \tau)$ where $\tau=\{\mathbb{N}, \phi,\{1\}\}$ with two ideals $I=\{\phi\}$ and $J$ is the

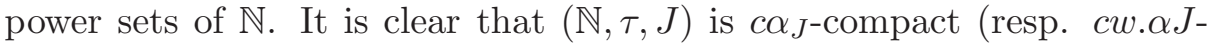
compact and $c w . J \alpha$-compact) but $(\mathbb{N}, \tau, I)$ is not $c \alpha_{I}$-compact (resp. not $c w . \alpha I$-compact and not $c w \cdot I \alpha$-compact), since $\{\{1, n\}: n \in \mathbb{N}\}$ is $\alpha_{I}$-open (resp. $w . \alpha I$-open and $w . I \alpha$-open) cover for $\mathbb{N}$ which has no finite subfamily $\{\{1, n\}: n=1,2, \cdots, r\}$ such that $\mathbb{N}-\cup\{\{1, n\}: n=$ $1,2, \cdots, r\} \in I$.

Proposition 17. If $A$ and $B$ are two c $\alpha_{I}$-compact subsets of an ideal topological space $(X, \tau, I)$, then $A \cup B$ is $c \alpha_{I}$-compact. 
Proof. Let $\left\{U_{\lambda}: \lambda \in \Lambda\right\}$ be an $\alpha_{I^{-}}$open cover for $A \cup B$, then it is an $\alpha_{I}$-open cover for both $A$ and $B$. So, there is a finite subsets $\Lambda_{1}$ and $\Lambda_{2}$ of $\Lambda$ such that $A-\cup\left\{U_{\lambda}: \lambda \in \Lambda_{1}\right\} \in I$ and $B-\cup\left\{U_{\lambda}: \lambda \in \Lambda_{2}\right\} \in I$. Implies, $\left(A-\cup\left\{U_{\lambda}: \lambda \in \Lambda_{1}\right\}\right) \cup\left(B-\cup\left\{U_{\lambda}: \lambda \in \Lambda_{2}\right\}\right) \in I$. Hence, $(A \cup B)-\left(\left(\cup\left\{U_{\lambda}: \lambda \in\right.\right.\right.$ $\left.\left.\left.\Lambda_{1}\right\}\right) \cup\left(\cup\left\{U_{\lambda}: \lambda \in \Lambda_{2}\right\}\right)\right) \subseteq\left(A-\cup\left\{U_{\lambda}: \lambda \in \Lambda_{1}\right\}\right) \cup\left(B-\cup\left\{U_{\lambda}: \lambda \in \Lambda_{2}\right\}\right) \in I$. Therefore, $(A \cup B)$ is $c \alpha_{I}$-compact.

In similar way, we can show that the union of two cw. $\alpha I$-compact (resp. $c w \cdot I \alpha$-compact) subsets of an ideal topological space $(X, \tau, I)$ is $c w \cdot \alpha I$-compact (resp. cw.I $\alpha$-compact) .

Remark 18. The intersection of two $c \alpha_{I}$-compact (resp. $c w . \alpha I$-compact and $c w \cdot I \alpha$-compact) subsets of an ideal topological space $(X, \tau, I)$ need not be $c \alpha_{I}$-compact (resp. cw. $\alpha I$-compact and $c w \cdot I \alpha$-compact) .

For example;

Consider the ideal topological space $(X, \tau, I)$, where $X=\mathbb{N} \cup\{-1,0\}$ with the indiscrete topology and $I=I_{f}=\{U \subseteq \mathbb{N}: U$ is a finite set $\}$. Let $A=$ $\mathbb{N} \cup\{0\}$ and $B=\mathbb{N} \cup\{-1\}$. Then $A$ is $c \alpha_{I}$-compact (resp. cw. $\alpha I$-compact and $c w . I \alpha$-compact) subsets of $X$, since every cover $\left\{U_{\lambda}: \lambda \in \Lambda\right\}$ for $A$ by $\alpha_{I^{-}}$-open (resp. $w . \alpha I$-open and $w \cdot I \alpha$-open) subsets of $X$ has at least one element $U_{\lambda_{0}}$. So $A-U_{\lambda_{0}}$ is a finite subset of $\mathbb{N}$.

Hence, $A$ is $c \alpha_{I}$-compact (resp. $c w \cdot \alpha I$-compact and $c w \cdot I \alpha$-compact) set. Similarly, $B$ is $c \alpha_{I}$-compact (resp. $c w \cdot \alpha I$-compact and $c w \cdot I \alpha$-compact) .

But $A \cap B=\mathbb{N}$ is not $c \alpha_{I}$-compact (resp. not $c w \cdot \alpha I$-compact and not $c w \cdot I \alpha$-compact), since the $\alpha_{I}$-open (resp. $w \cdot \alpha I$-open and $w \cdot I \alpha$-open) cover $\{\{n\}: n \in \mathbb{N}\}$ has no finite subcover $\{\{n\}: n=1,2, r\}$ such that $\mathbb{N}-\cup\{\{n\}$ : $n=1,2,, r\} \in I$.

Remark 19. $c \alpha_{I}$-Compactness (resp. cw. $\alpha I$-compactness and $c w \cdot I \alpha$ compactness) is not hereditary property. See the example of Remark $18, X$ is $c \alpha_{I}$-compact (resp. $c w . \alpha I$-compact and $c w \cdot I \alpha$-compact) space but $\mathbb{N}$ is not.

Proposition 20. $\alpha_{I}$-Closed (resp. w. $\alpha I$-closed and w.I $\alpha$-closed) subset of an $c \alpha_{I^{-}}$-compact (resp. cw. $\alpha I$-compact and $c w . I \alpha$-compact) space is $c \alpha_{I^{-}}$ compact (resp. cw. $\alpha I$-compact and cw.I $\alpha$-compact) .

Remark 21. [12]

(i) If $f:(X, \tau, I) \rightarrow(Y, \sigma)$ is a function, then $f(I)=\{f(J): J \in I\}$ is an ideal on $Y$.

(ii) If $f:(X, \tau) \rightarrow(Y, \sigma, J)$ is an injection function, then $f^{-1}(J)=\{f(B)$ : $B \in J\}$ is an ideal on $X$. 
Definition 22. A function $f:(X, \tau, I) \rightarrow(Y, \sigma, J)$ is said to be

(i) $\alpha_{I^{-o p e n}}$ (resp. w. $\alpha I$-open and $w . I \alpha$-open) function if $f(A)$ is $\alpha_{J^{-o p e n}}$ (resp. $w . \alpha J$-open and $w . J \alpha$-open) set in $Y$ for each $\alpha_{I^{-o p e n}}$ (resp. $w . \alpha I$ open and $w . I \alpha$-open) set $A$ in $X$.

(ii) $\alpha_{I^{-}}$irresolute (resp. w. $\alpha I$ - irresolute and $w . I \alpha$ - irresolute) function if $f^{-1}(B)$ is $\alpha_{I}$-open (resp. $w . \alpha I$-open and $w . I \alpha$-open) set in $X$ for each $\alpha_{J}$-open (resp. w. $\alpha J$-open and $w . J \alpha$-open) set $B$ in $Y$.

Proposition 23. If $f:(X, \tau, I) \rightarrow(Y, \sigma, f(I))$ is an $\alpha_{I^{-}}$irresolute (resp. $w . \alpha I$ - irresolute and w.I $\alpha$ - irresolute) surjection function and $(X, \tau, I)$ is $c \alpha_{I}$ - compact (resp. cw. $\alpha I$ - compact and cw.I $\alpha$ - compact), then $(Y, \sigma, f(I))$ is $c \alpha_{f(I)}$ - compact (resp. cw. $\alpha f(I)$ - compact and cw.f(I) $\alpha$ - compact) .

Corollary 24. If $f:\left(X, \tau, f^{-1}(J)\right) \rightarrow(Y, \sigma, J)$ is $\alpha_{f^{-1}(J)^{-o p e n ~}}$ (resp. $w \cdot \alpha f^{-1}(J)$-open and $w \cdot f^{-1}(J) \alpha$-open) bijection function and $(Y, \sigma, J)$ is $c \alpha_{J^{-}}$ compact (resp. cw. $\alpha J$-compact and cw.J $\alpha$-compact) space, then $\left(X, \tau, f^{-1}(J)\right)$ is $c \alpha_{f^{-1}(J)}$ - compact (resp. cw. $\alpha f^{-1}(J)$ - compact and cw. $f^{-1}(J) \alpha$-compact)

Definition 25. A subset $A$ of an ideal topological space $(X, \tau, I)$ is said to be countably $\alpha_{I}$-compact (resp. countably $w . \alpha I$-compact and countably $w . I \alpha$ compact) if for every countable cover $\left\{U_{n}: n \in \mathbb{N}\right\}$ of $A$ by $\alpha_{I^{-}}$open (resp. $w . \alpha I$-open and $w . I \alpha$-open) sets of $X$, there exists a finite subset $\mathbb{N}_{0}$ of $\mathbb{N}$ such that $A-\cup\left\{U_{n}: n \in \mathbb{N}_{0}\right\} \in I$. An ideal topological space $(X, \tau, I)$ is said to be countably $\alpha_{I}$-compact (resp. countably $w \cdot \alpha_{I}$-compact and countably $w \cdot I \alpha$ compact) if $X$ is countably $\alpha_{I}$-compact (resp. countably $w \cdot \alpha I$-compact and countably $w . I \alpha$-compact) as a subset.

Proposition 26. Every c $\alpha_{I}$-compact (resp. cw. $\alpha I$-compact and $c w . I \alpha$ compact) space is countably $\alpha_{I}$-compact (resp. countably w. $\alpha I$-compact and countably w.I $\alpha$-compact) .

The implication of Proposition 26, is not reversible as the following example shows.

Example 27. Consider the ideal topological space $(\mathbb{R}, \tau, I)$ where $\mathbb{R}$ is the set of all real numbers, $\tau=P(\operatorname{Irr}) \cup\{Q \cup A: A \subseteq \operatorname{Irr}\}$ and $I=\{\phi\}$, such that $\operatorname{Irr}$ is the set of irrational numbers, $Q$ is the set of rational numbers and $P(\operatorname{Irr})$ is the set of all subsets of the irrational numbers.

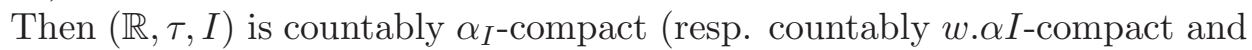
countably $w . I \alpha$-compact), since every countable cover of $\mathbb{R}$ by $\alpha_{I}$-open (resp. $w . \alpha I$-open and $w . I \alpha$-open $)$ sets is a finite cover. But $(\mathbb{R}, \tau, I)$ is not $c \alpha_{I}$-compact 
(resp. not $c w \cdot \alpha I$-compact and not $c w \cdot I \alpha$-compact), since $\{Q \cup\{x\}: x \in I r r\}$ is an $\alpha_{I}$-open (resp. $w . \alpha I$-open and $w . I \alpha$-open) cover for $\mathbb{R}$ which has no finite subcover $M$ such that $\mathbb{R}-M \in I$.

Remark 28. The concepts $I$-compactness and countably $\alpha_{I}$-compactness (resp. countably $w \cdot \alpha I$-compactness and countably $w \cdot I \alpha$-compactness) are independent as the following examples show.

(i) See Example 27, $(\mathbb{R}, \tau, I)$ is countably $\alpha_{I}$-compact (resp. countably $w . \alpha I$ compact and countably $w . I \alpha$-compact) space which is not $I$-compact.

(ii) See Example 16, the ideal topological space $(\mathbb{N}, \tau, I)$, where $\tau=\{\mathbb{N}, \phi,\{1\}\}$ and $I=\{\phi\}$, is an $I$-compact space which is not countably $\alpha_{I}$-compact (resp. not countably $w \cdot \alpha I$-compact and not countably $w \cdot I \alpha$-compact).

\section{Connectedness with Respect to an $\alpha_{I}$-Open, w. $\alpha I$-Open and $w . I \alpha$-Open Set}

In this section, we will introduce new types of connectedness in ideal topological spaces by using the concept of $\alpha_{I}$-open, $w . \alpha I$-open and $w$.I $\alpha$-open set. Some properties of these types are studied.

Definition 29. [5] An ideal topological space $(X, \tau, I)$ is said to be *hyperconnected if $A$ is $\tau^{*}$-dense $\left(c l^{*}(A)=X\right)$ for every nonempty open subset $A$ of $X$.

Definition 30. [2] An ideal topological space $(X, \tau, I)$ is said to be $I \star$ hyperconnected if $X-c l^{*}(A) \in I$ for every nonempty open subset $A$ of $X$.

Definition 31. An ideal topological space $(X, \tau, I)$ is said to be $\alpha_{I^{\star} \text { - }}$ hyperconnected (resp. w. $\alpha \star$-hyperconnected and $w \cdot I \alpha \star$-hyperconnected) if $X-c l^{*}(A) \in I$ for every nonempty $\alpha_{I}$-open (resp. $w \cdot \alpha I$-open and $w \cdot I \alpha$-open) subset $A$ of $X$.

It is clear that every $\alpha_{I \star} \star$-hyperconnected space is $w \cdot \alpha I \star$-hyperconnected and every $w \cdot \alpha I \star$-hyperconnected space is $w \cdot I \alpha \star$-hyperconnected.

Remark 32. The following properties hold for an ideal topological space $(X, \tau, I)$;

(i) Every $\star$-hyperconnected is an $I \star$-hyperconnected, not conversely [2].

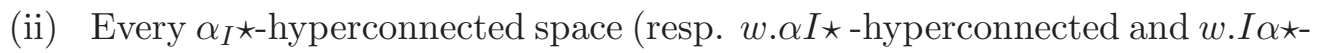
hyperconnected) is an $I \star$-hyperconnected, not conversely. 
(iii) The concepts $\alpha_{I} \star$-hyperconnectedness (resp. $w . \alpha I \star$-hyperconnectedness and $w . I \alpha \star$-hyperconnectedness) and $\star$-hyperconnectedness are independent.

Example 33. Consider the ideal topological space $(X, \tau, I)$, where $X$ is any set has more than one element with $\tau=\{X, \phi,\{x\}\}$ and $I=P(X)$. Then $X$ is $I \star$-hyperconnected, $\alpha_{I \star}$-hyperconnected, $w \cdot \alpha I \star$-hyperconnected and $w \cdot I \alpha \star$-hyperconnected which is not $\star$-hyperconnected.

Example 34. Consider the ideal topological space $(X, \tau, I)$, where $X=$ $\{1,2,3\}$ with the indiscrete topology and $I=\{\phi,\{1\},\{2\},\{1,2\}\}$. Then $X$ is $\star$-hyperconnected and $I \star$-hyperconnected which is not $\alpha_{I} \star$-hyperconnected , not $w \cdot \alpha I \star$-hyperconnected and not $w \cdot I \alpha \star$-hyperconnected) .

Proposition 35. For any ideal topological space $(X, \tau, I)$

(i) If $I=\{\phi\}$, then the concepts $\star$-hyperconnectedness and $I \star$-hyperconnectedness are the same [2].

(ii) If $I=\{\phi\}$ and every open subset of $X$ is closed, then the concepts $\star$-hyperconnectedness, $I \star$-hyperconnectedness, $\alpha_{I \star}$-hyperconnectedness, $w . \alpha I \star$-hyperconnectedness and w.I $\alpha \star$-hyperconnectedness are equivalent.

Proposition 36. If an ideal topological space $(X, \tau, I)$ is $\alpha_{I}$-hyperconnectedness (resp. $w . \alpha I \star-h y p e r c o n n e c t e d n e s s$ and $w . I \alpha \star$-hyperconnectedness) then, $X-c l^{*}(A) \in I$ for every nonempty $\alpha$-open (resp. $\alpha I$-open and $I \alpha$-open) subset $A$ of $X$.

The implication in Proposition 36 is not reversible as the following example shows;

Example 37. Consider the ideal topological space $(X, \tau, I)$ where $X=$ $\{a, b, c\}, \tau=\{X, \phi,\{a\}\}$ and $I=\{\phi,\{b\},\{c\},\{b, c\}\}$. Then, $X-c l^{*}(A) \in I$ for every nonempty $\alpha$-open (resp. $\alpha I$-open and $I \alpha$-open) subset $A$ of $X$ but $X-c l^{*}(\{b, c\}) \notin I$ where $\{b, c\}$ is $\alpha_{I}$-open (resp. $w . \alpha I$-open and $w . I \alpha$-open) subset of $X$.

Proposition 38. For any ideal topological space $(X, \tau, I)$, the following statements are equivalent;

(i) $X$ is $\alpha_{I^{\star}}$-hyperconnectedness (resp. w. $\alpha I \star$-hyperconnectedness and $w . I \alpha \star$-hyperconnectedness) .

(ii) $\operatorname{int}^{*}(B) \in I$ for every proper $\alpha_{I}$-closed (resp. w. $\alpha I$-closed and w.I $\alpha$ closed) subset of $X$. 
Definition 39. Let $(X, \tau, I)$ be an ideal topological space and $A$ is a subset of $X$. An $\alpha_{I}$-closure (resp. w. $\alpha I$-closure and $w \cdot I \alpha$-closure) of $A$ is the smallest $\alpha_{I}$-closed (resp. $w \cdot \alpha I$-closed and $w \cdot I \alpha$-closed) set containing $A$, denoted by $\alpha_{I}-\operatorname{cl}(A)$ (resp. $w \cdot \alpha I-\operatorname{cl}(A)$ and $\left.w \cdot I \alpha-\operatorname{cl}(A)\right)$.

Definition 40. A nonempty subsets $A$ and $B$ of an ideal topological space $(X, \tau, I)$ are $\alpha_{I}$-separated (resp. $w . \alpha I$-separated and $w \cdot I \alpha$-separated) sets if $\alpha_{I}-\operatorname{cl}(A) \cap B=\phi=A \cap c l_{\alpha}(B)$ (resp. $w . \alpha I-\operatorname{cl}(A) \cap B=\phi=A \cap \alpha I-\operatorname{cl}(B)$ and $w \cdot I \alpha-\operatorname{cl}(A) \cap B=\phi=A \cap I \alpha-\operatorname{cl}(B))$.

Definition 41. A subset $A$ of an ideal topological space $(X, \tau, I)$ is $\alpha_{I^{-}}$ separated (resp. $w . \alpha I$-separated and $w . I \alpha$-separated) connected if it cannot be written as a union of two $\alpha_{I}$-separated (resp. w. $\alpha I$-separated and $w . I \alpha$ -separated) sets in $X$. An ideal topological space $(X, \tau, I)$ is said to be $\alpha_{I^{-}}$ separated (resp. $w . \alpha I$-separated and w.I $\alpha$-separated) connected if $X$ is $\alpha_{I^{-}}$ separated (resp. $w . \alpha I$-separated and $w \cdot I \alpha$-separated) connected as a subset.

Remark 42. Let $(X, \tau, I)$ be an ideal topological space. If $A$ and $B$ are nonempty disjoint subsets of $X$ such that $A$ is $\alpha$-open (resp. $\alpha I$-open and $I \alpha$ - open) set and $B$ is $\alpha_{I}$-open (resp. $w . \alpha I$-open and $w \cdot I \alpha$-open) set, then $A$ and $B$ are $\alpha_{I}$-separated (resp. $w . \alpha I$-separated and $w \cdot I \alpha$-separated) sets.

Remark 43. If an ideal topological space $(X, \tau, I)$ is $\alpha_{I}$-separated (resp. $w . \alpha I$-separated and $w \cdot I \alpha$-separated) connected then, $(X, \tau)$ is connected.

The implication in Remark 43 is not reversible as the following example shows;

Example 44. Consider the ideal topological space $(\mathbb{N}, \tau, I)$ where $\tau=$ $\{\mathbb{N}, \phi,\{1\}\}$ and $I$ is the power set of $\mathbb{N}$. It is clear that $(\mathbb{N}, \tau)$ is connected space but $(\mathbb{N}, \tau, I)$ is not $\alpha_{I}$-separated connected (resp. not $w . \alpha I$-separated connected and not $w \cdot I \alpha$-separated connected) .

Proposition 45. Let $Y$ be an open subset of an ideal topological space $(X, \tau, I)$ and $A$ is a subset of $X$. If $A$ is an $\alpha_{I}$-open (resp. w. $\alpha I$-open and w.I $\alpha$ -open) subset of $X$, then $A \cap Y$ is $\alpha_{I Y}$-open (resp. w. $\alpha I_{Y}$-open and w.I $I_{Y} \alpha$ -open) subset of $Y$.

The implication of Proposition 45 is not reversible, in general. As the following example shows;

Example 46. Consider the ideal topological space $(X, \tau, I)$ where $X=$ $\{a, b, c, d\}, \tau=\{X, \phi,\{a, c\}\}$ and $I=\{\phi,\{a\},\{b\},\{a, b\}\}$. Take $Y=\{a, c\}$ and $A=\{a, d\}$. 
Proposition 47. Let $A \subseteq Y$ and $Y$ be an open subset of an ideal topological space $(X, \tau, I) . A$ is $\alpha_{I Y}$-open (resp. $w \cdot \alpha I_{Y}$-open and $w \cdot I_{Y} \alpha$-open) subset of $Y$ if and only if it is $\alpha_{I}$-open (resp. $w . \alpha I$-open and $w . I \alpha$-open) subset of $X$.

Proposition 48. If $A \subseteq Y$ and $Y$ be an open subset of an ideal topological space $(X, \tau, I)$ then, $\alpha_{I Y} c l_{Y}(A)=\alpha_{I} c l(A) \cap Y$.

Proof. First, to prove $\alpha_{I Y} c l_{Y}(A) \subseteq \alpha_{I} c l(A) \cap Y$.

Let $x \notin \alpha_{I} c l(A) \cap Y$ implies, $x \in X-\alpha_{I} c l(A)$ and by Proposition 45, $\left(X-\alpha_{I} c l(A)\right) \cap Y$ is an $\alpha_{I Y}$-open set in $Y$ containing $x$. So, $A \subseteq Y-((X-$ $\left.\left.\alpha_{I} c l(A)\right) \cap Y\right)=\alpha_{I} c l(A) \cap Y$.

Now, if $\alpha_{I} \operatorname{cl}(A) \cap Y$ is the smallest $\alpha_{I Y}$-closed set in $Y$ containing $A$ then the proof is over.

If not, then there exists an $\alpha_{I Y}$-closed set, say $B$, in $Y$ containing $A$. So, $B \cap\left(\alpha_{I} \operatorname{cl}(A) \cap Y\right)$ is an $\alpha_{I Y}$-closed set in $Y$ containing $A$ and $x \notin B \cap\left(\alpha_{I} \operatorname{cl}(A) \cap\right.$ $Y)=B$.

Hence, $x \notin \alpha_{I Y} c l_{Y}(A)$.

Second, to prove $\alpha_{I} c l(A) \cap Y \subseteq \alpha_{I Y} c l_{Y}(A)$.

Let $x \notin \alpha_{I Y} c l_{Y}(A)$ implies, $x \in Y-\alpha_{I Y} c l_{Y}(A)$ and by Proposition 47, $Y-\alpha_{I Y} c l_{Y}(A)$ is an $\alpha_{I}$-open set in $X$ containing $x$. So, $A \subseteq X-(Y-$ $\left.\alpha_{I Y} c l_{Y}(A)\right)=\alpha_{I Y} c l_{Y}(A) \cup(X-Y)$.

Now, if $\alpha_{I Y} c l_{Y}(A) \cup(X-Y)$ is the smallest $\alpha_{I}$-closed set in $X$ containing $A$ then the proof is over.

If not, then there exists an $\alpha_{I}$-closed set, say $B$ in $X$ containing $A$. So, $B \cap\left(\alpha_{I Y} c l_{Y}(A) \cup(X-Y)\right)$ is an $\alpha_{I}$-closed set in $X$ containing $A$ and $x \notin$ $B \cap\left(\alpha_{I Y} c l_{Y}(A) \cup(X-Y)\right)=B$.

Hence, $x \notin \alpha_{I} \operatorname{cl}(A)$.

In similar way we can prove that where $A \subseteq Y$ and $Y$ be an open subset of an ideal topological space $(X, \tau, I)$ then, $w \cdot \alpha I_{Y} c l_{Y}(A)=w \cdot \alpha I c l(A) \cap Y$ (resp. $\left.w \cdot I_{Y} \alpha c l_{Y}(A)=w \cdot \operatorname{I} \alpha c l(A) \cap Y\right)$.

Proposition 49. Let $A$ and $B$ are two subsets of $Y$ and $Y$ is an open subset of an ideal topological space $(X, \tau, I)$. The following are equivalent;

(i) $A$ and $B$ are $\alpha_{I Y}$-separated (resp. w. $\alpha I_{Y}$-separated and w.I $I_{Y} \alpha$-separated in $Y$.

(ii) $A$ and $B$ are $\alpha_{I}$-separated (resp. w. $\alpha I$-separated and w.I $\alpha$-separated in $X$. 
Proof. The proof follows immediately from Proposition 48 .

Proposition 50. An ideal topological space $(X, \tau, I)$ is $\alpha_{I^{-}}$separated (resp. w. $\alpha I$ - separated and w.I $\alpha$ - separated) connected space if and only if it is cannot be written as a disjoint union of a nonempty $\alpha$-open (resp. $\alpha I$-open and $I \alpha$-open) and $\alpha_{I}$-open (resp. w. $\alpha I$-open and $w \cdot I \alpha$-open) subsets of $X$.

Proposition 51. Let $(X, \tau, I)$ be an ideal topological space and $A$ is an open subset of $X$. If $A$ is $\alpha_{I^{-}}$separated (resp. w. $\alpha I$ - separated and w.I $\alpha$ separated) connected subset of $X$ and $H, G$ are $\alpha_{I^{-}}$separated (resp. w. $\alpha I$ separated and w.I $\alpha$ - separated) subsets of $X$ with $A \subseteq H \cup G$, then either $A \subseteq H$ or $A \subseteq G$.

Proof. Suppose $A \cap H$ and $A \cap G$ are nonempty. Since $A \subseteq H \cup G$, then $A=(A \cap H) \cup(A \cap G)$. So, $\alpha_{I} c l(A \cap H) \cap(A \cap G) \subseteq \alpha_{I} c l(H) \cap G=\phi$ and $(A \cap H) \cap c_{\alpha}(A \cap G) \subseteq H \cap c l_{\alpha}(G)=\phi$.

Then, $\left(\alpha_{I} c l(A \cap H) \cap A\right) \cap(A \cap G)=\phi$ and $(A \cap H) \cap\left(c_{\alpha}(A \cap G) \cap A\right)=\phi$.

By Proposition 48, $\left(\alpha_{I A} c l(A \cap H)\right) \cap(A \cap G)=\phi$ and $(A \cap H) \cap\left(c l_{\alpha A}(A \cap G)\right)=$ $\phi$.

Then, $A$ is not $\alpha_{I}$-separated connected. This is a contradiction. Thus, either $A \cap H$ or $A \cap G$ are empty. Implies, either $A \subseteq H$ or $A \subseteq G$.

In similar way, we can prove the implication in Proposition 51 where $H, G$ are $w . \alpha I$ - separated (resp. $w . I \alpha$ - separated) subsets of $X$.

Proposition 52. Let $A$ and $B$ are two $\alpha_{I^{-}}$separated (resp. w. $\alpha I$ separated and $w . I \alpha$ - separated) subsets of an ideal topological space $(X, \tau, I)$. If $C$ and $D$ are two nonempty subsets of $X$ such that $C \subseteq A$ and $D \subseteq B$, then $C$ and $D$ are also $\alpha_{I^{-}}$separated (resp. $w . \alpha I$ - separated and w.I $\alpha$-separated)

Proposition 53. The continuous image of an $\alpha_{I^{-}}$separated (resp. w. $\alpha I$ - separated and $w \cdot I \alpha$ - separated) connected space is connected.

Proof. Follow from Remark 43.

Proposition 54. If $A$ is an $\alpha_{I^{-}}$separated (resp. w. $\alpha I$ - separated and $w . I \alpha$ - separated) connected subset of an $\alpha_{I^{-}}$separated (resp. w. $\alpha I$ - separated and $w . I \alpha$ - separated) connected ideal space $(X, \tau, I)$ such that $X-A$ is the union of two $\alpha_{I^{-}}$separated (resp. $w . \alpha I$ - separated and w.I $\alpha$ - separated) sets $B$ and $C$, then $A \cup B$ and $A \cup C$ are $\alpha_{I^{-}}$separated (resp. w. $\alpha I$ - separated and $w \cdot I \alpha$ - separated) connected. 
Proof. Suppose $A \cup B$ is not $\alpha_{I}$-separated connected. Then there exist two nonempty $\alpha_{I}$-separated sets $G$ and $H$ such that $A \cup B=G \cup H$.

Since $A$ is $\alpha_{I}$-separated connected, $A \subset G \cup H$, by Proposition 51, either $A \subset G$ or $A \subset H$. Suppose $A \subset G$ implies that $A \cup B \subset G \cup B$.

Since $A \cup B=G \cup H$ then $G \cup H \subset G \cup B$. Hence, $H \subset B$.

Since $B$ and $C$ are $\alpha_{I}$-separated, by Proposition 52, $H$ and $C$ are also $\alpha_{I}$-separated. Thus $H$ is $\alpha_{I}$-separated from $G$ as well as $C$.

Now, $\alpha_{I} c l(H) \cap(G \cup C)=\left(\alpha_{I} c l(H) \cap G\right) \cup\left(\alpha_{I} c l(H) \cap C\right)=\phi$ and $H \cap$ $c l_{\alpha}(G \cup C)=\left(H \cap c l_{\alpha}(G)\right) \cup\left(H \cap c l_{\alpha}(C)\right)=\phi$.

Therefore, $H$ is $\alpha_{I}$-separated from $G \cup C$.

Since $X-A=B \cup C, X=A \cup(B \cup C)=(A \cup B) \cup C=(G \cup H) \cup C=$ $H \cup(G \cup C)$. Thus, $X$ is not $\alpha_{I}$-separated connected. This is a contradiction. Similar, if $A \subset H$ we will get a contradiction. Hence, $A \cup B$ is $\alpha_{I \text {-separated }}$

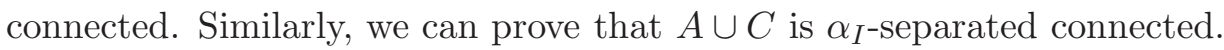

In similar way, we can prove that where $A$ is an $w . \alpha I$ - separated (resp. $w . I \alpha$ - separated) connected subset of an $w . \alpha I$ - separated (resp. $w . I \alpha$ - separated) connected ideal space $(X, \tau, I)$ such that $X-A$ is the union of two $w \cdot \alpha I$ separated (resp. w.I $\alpha$ - separated) sets $B$ and $C$, then $A \cup B$ and $A \cup C$ are $w . \alpha I$ - separated (resp. $w . I \alpha$ - separated) connected.

Proposition 55. Let $(X, \tau, I)$ be an ideal space. If the union of two $\alpha_{I^{-}}$ separated (resp. w. $\alpha I$ - separated and $w . I \alpha$ - separated) sets is an $\alpha$-closed (resp. $\alpha I$-closed and $I \alpha$-closed) set, then one set is $\alpha$-closed (resp. $\alpha I$-closed and $I \alpha$-closed) and the other is $\alpha_{I^{-}}$closed (resp. w. $\alpha I$-closed and w.I $\alpha$-closed)

Proof. Let $A$ and $B$ two $\alpha_{I}$-separated sets such that $A \cup B$ is $\alpha$-closed. Then $\alpha_{I} c l(A) \cap B=A \cap c_{\alpha}(B)=\phi$.

Since $A \cup B$ is $\alpha$-closed, $A \cup B=c_{\alpha}(A \cup B)$.

Now, $A \subset A \cup B$ implies that $\alpha_{I} c l(A) \subset \alpha_{I} c l(A \cup B) \subset c l_{\alpha}(A \cup B)=A \cup B$ and so $\alpha_{I} \operatorname{cl}(A)=\alpha_{I} c l(A) \cap(A \cup B)=A$.

Hence, $A$ is $\alpha_{I \text {-closed. }}$.

Also, $c l_{\alpha}(B)=c l_{\alpha}(B) \cap c l_{\alpha}(A \cup B)=c l_{\alpha}(B) \cap(A \cup B)=B$.

Hence, $B$ is $\alpha$-closed.

In similar way, we can prove that where the union of two $w \cdot \alpha I$ - separated (resp. $w . I \alpha$ - separated) sets is an $\alpha I$-closed (resp. $I \alpha$-closed) set, then one set is $\alpha I$-closed (resp. I $\alpha$-closed) and the other is $w . \alpha I$ - closed (resp. w.I $\alpha$ closed) . 


\section{Conclusion and Future Work}

In this paper begins with a brief survey of the notion of ideal in topological spaces introduced by K. Kuratowski [8], V. Vaidyanathaswamy [14] and D. Jankovic, T. R. Hamlett [7].

We recall different types of collections of sets in topological spaces $(X, \tau)$ and in an ideal topological spaces $(X, \tau, I)$, like $\alpha$-open sets [11], $\alpha I$-open sets [6], $I \alpha$-open sets [3], $\alpha_{I}$-open set, $w . \alpha I$-open set and $w \cdot I \alpha$-open set [1].

The authors studied some properties of an $\alpha_{I}$-open (resp. w. $\alpha I$-open and $w . I \alpha$-open) set in ideal topological spaces, like compactness and connectedness.

In particular, the authors investigate the relationship between the different types of compactness (resp. connectedness) that introduced in this paper.

Moreover, several examples are given to indicate the connection between these types. It also gives some conditions to make these notions equivalence and prove some properties of these concepts.

Finally, by using the notion $\alpha_{I}$-open (resp. w. $\alpha I$-open and $w \cdot I \alpha$-open) set, the authors introduced some types of continuity functions in ideal topological spaces. The affecting of these functions on some types of compactness investigated.

The concept $\alpha_{I}$-open (resp. w. $\alpha I$-open and $w \cdot I \alpha$-open) set in an ideal topological space with related properties help to study new types of continuity and separation axioms.

\section{References}

[1] M. E. Abd El-Monsef, A. A. Nasef, A. E. Radwan and R. B. Esmaeel, On $\alpha$-open sets with respect to an ideal, Journal of Advanced Studies in Topology, 5(3) (2014), 1-9.

[2] M. E. Abd El-Monsef, A. A. Nasef, A. E. Radwan, F. A. Ibrahem and R. B. Esmaeel, Some properties of semi-open sets with respect to an ideal, (submitted).

[3] M. E. Abd El-Monsef, A. E. Radwan and A. I. Nasir, Some generalized forms of connectedness in ideal topological space, Archives Des Sciences, Vol 66, No. 3; Mar (2013), 334-342.

[4] J. Dontchev, M. Ganster and D. Rose, Ideal resolvability, Topology and its Appl. 93(1991), 1-16. 
[5] E. Ekici and T. Noiri, ^-Hyperconnected ideal topological spaces, Analele Stiintifice Aie Universitatii Matematica (2012), 121- 129.

[6] E. Hatir and T. Noiri, On decomposition of continuity via idealization, Acta Math. Hungar., 96(4)(2002), 341- 349.

[7] D. Jankovic and T. R. Hamlett, New topologies from old via ideals, Amer. Math. Monthly, 97(1990), 295- 310.

[8] K. Kuratowski, Topology, Vol I. New York: Academic Press, (1933).

[9] S. N. Maheshwari and S. S. Thakur, On $\alpha$-compact spaces, Bulletin of The Institute of Mathematics, Academia Sinice, Vol. 13, No. 4, Dece. (1985), $341-347$.

[10] A. A. Nasef, Ideals in general topology, Ph. D. Thesis, Tanta University, Egypt, 1992.

[11] O. Njastad, On some classes of nearly open sets, Pacific J. Math. 15(1965), 961- 970 .

[12] R. L. Newcomb, Topologies which are compact modulo an ideal, Ph. D. dissertation, Univ. of Cal. At Santa Barbara, (1967).

[13] D. V. Rancin, Compactness modulo an ideal, Soviet Math. Dokl., 13(1)(1972), 131- 147.

[14] V. Vaidyanathaswamy, The localization theory in set topology, Proc. Indian Acad. Sci. 20(1945), 51- 61. 